\title{
The making of integrated care
}

Over the four decades that followed the creation of the NHS, British diabetes management slowly spread outside the hospital. During the late 1940s and the 1950s, clinicians first co-operated with local government public health doctors to extend the reach of surveillance and education. From the late 1960s onwards, GPs assumed roles as coordinators and providers of care in the community, developing systems of disease management on their own and in collaboration with specialists. Although individual GPs moved into diabetes care for a range of reasons, by the 1980s professional bodies like the RCGP had connected diabetes management to professional projects. The promotion of diabetes management formed part of efforts to enhance GP responsibility for preventive medicine, with understandings of secondary and tertiary prevention recasting GP diabetes care as an innovative form of risk management.

The re-spatialisation of care, together with a growing emphasis on surveillance and blood glucose control, raised questions for clinicians and GPs involved in diabetes management. The first was how doctors involved in new schemes could prevent patients falling through the gaps between different sites of oversight; the second - serving as a mirror image - was how practitioners could avoid unnecessary duplication of labour. In short, how could care be co-ordinated? Furthermore, in the context of political and professional anxiety about professional competence, both specialists and GPs asked how they could ensure that surveillance and treatment would be of sufficient quality. In other words, they asked what counted as good care, and how standards could be maintained within innovative organisational arrangements.

This chapter examines how creators of novel systems for diabetes management sought to answer these questions at a local level. Concentrating on the use of recall systems, patient records, and care protocol, 
it explores how practitioners drew upon pre-existing bureaucratic cultures of medicine, finding solutions to their concerns in the combination of instruments originally intended for refining patient surveillance. All three devices were initially designed to ensure timely review, to store and provide longitudinal data, to divide responsibility for oversight, and to communicate what tasks had - or had not - been performed. The deployment of such instruments, however, also required the codification of 'good care' (to decide, for instance, when patients should be seen, or what tests should be performed), and inherently regulated the temporality and content of professional work. In fact, over time, the designers of new instruments articulated the politics inherent in these apparently inert 'objects', explicitly framing them as attempts to secure good care through the management of professional labour. ${ }^{1}$

Historically, doctors had perceived almost any formal control over their practice as a threat to professional freedom. Yet in the case of systematic and integrated care, references to instruments' 'built-in reminders' implied a mistrust of unaided professional expertise, and clearly articulated benefits to managed clinical decision-making. To be sure, the 'disciplinary' mesh that regulated the time, space, activity, and visibility of medical practice was far looser than that classically designed in the prison or factory. ${ }^{2}$ Similarly, tools like protocols and records could build on routines that already existed, or - once mobilised - might enable practitioners to focus on complex tasks requiring more tacit skill. ${ }^{3}$ Nonetheless, the devices discussed here structured and divided medical labour, determined rhythms of activity, recorded work, and subjected professionals to review in ways hitherto unseen. They turned previously implicit and loose clinical norms into explicit guidance. In other words, these new combinations of concepts, instruments, and actors formed what, following historians and sociologists of science, we might call local 'organisational technologies' of quality, ones constructed to subject professionals - not just patients - to management. ${ }^{4}$

It would be easy to assume that the creation of such technologies resulted from Machiavellian politicking on behalf of mistrustful specialists and academics. As noted in Chapter 2, consultants had long been suspicious of GPs' capabilities. Moreover, the instruments used to regulate care emerged from traditional hierarchies of medicine. Indeed, in specific instances specialists pushed for new forms of work out of scepticism about GPs. Nonetheless, the new arrangements also reflected a shared sense within the diabetological community - and, evidently, within elite British medicine more broadly - that quality care depended 
upon bureaucratising instruments: tools that formally codified work processes and responsibilities and held up actions of team members for review. ${ }^{5}$ GPs themselves designed new systems, and once enrolled, all practitioners would find their work patterns reoriented, their responsibilities and actions recorded, and their performance reviewed.

As will be seen in Chapters 5 and 6, the local development of new models for managed, integrated care had implications for, and were tied into, national trends. This chapter, however, looks at the early experiments with professional management on a local level to assess the politics and mechanisms of their spread, and to trace the way in which programmes for diabetes drew upon, and provided a model for, the control of other chronic diseases.

Surveillance, patient-centred care, and managing temporality

As discussed in previous chapters, specialists after the 1960s increasingly emphasised the importance of close surveillance in diabetes management. Shifts in discussions of preventive medicine - triggered by new treatments, NHS reform, and epistemological and evidential change - made oversight more important for all practitioners.

These drives to surveillance found further support from renewed emphases on patient self-management. The 1970s and 1980s saw a marked increase in discussion of patient education and 'patientcentred' care. Textbooks, for instance, professed how 'one of the most important advances in diabetic care has been the recognition that the most important person in the Health Care Team ... is the diabetic.' As discussed at length elsewhere, the drivers for this focus in diabetes management were numerous. ${ }^{7}$ Political interest in patient rights, for instance, had grown since the $1960 \mathrm{~s}^{8}{ }^{8}$ Medical scandals, rising costs, and political critiques of medical power also contributed to moves to de-institutionalise patients and increase lay input into healthcare. ${ }^{9}$ Medical discourse more broadly had been slowly embracing questions of patient behaviour and social experience since the early 1920s, accelerating in the post-war period as more formal techniques of investigation were imported from sociology, psychology, and economics. ${ }^{10}$ In diabetes care itself, technological innovations were also crucial. The development of self-monitoring blood glucose machines and insulin pumps had promised to enhance the ability of patients to manage their own care with greater accuracy. ${ }^{11}$ Studies with these machines had 
delivered positive outcomes, and their use by selected patients found support amongst clinicians. ${ }^{12}$

With patients able to monitor their own physiologies and make relevant therapeutic adjustments to maintain control, it might be thought that clinical teams would relinquish observational duties. However, even the most optimistic doctor felt that pumps and self-monitoring had limits. The growth of patient-centred discourse thus involved a considerable discussion of education, as well as psychological and social support. ${ }^{13}$ Technological changes may have enhanced the possibility of self-care, but, handbooks suggested, 'a new responsibility falls upon the Health Care Team' if 'the patient is to accept responsibility for his or her own health care rather than rely on others. ${ }^{14}$ Equally, elite practitioners spoke of the need to offer continued social and psychological support to the patient, innovating with the use of psychologists and lay group therapies. ${ }^{15}$ They argued that making effective decisions required more than information, proposing that the patient needs someone to whom he or she can turn in order to gain advice. ${ }^{16}$ Moreover, there were concerns that patients could become demotivated by the lifelong grind of self-care and lack of guaranteed results: 'tomorrow never comes', as one leading practitioner put it. ${ }^{17}$ Therefore, specialists argued, an increased regularity of follow-up consultations would be beneficial: as well as improving biochemical surveillance, regular follow-up appointments would 'boos[t] the patient's morale and fortif $[y]$ his [sic] determination to continue self-care. ${ }^{18}$

This emphasis upon the regularity of oversight marked something of a shift from earlier discursive foci. Constant monitoring of disease had been a feature of specialist clinics since the 1920s, but popular handbooks and textbooks rarely discussed the timing of oversight. ${ }^{19}$ Even where authors made recommendations about regularity, their deliberations did not extend to active time management. ${ }^{20}$ Moreover, whilst hospitals commonly deployed appointment systems by the 1950s, they were not used in a consistent manner, either within or between clinics. ${ }^{21}$ Like other outpatient facilities, diabetic clinics block-booked patients for the start of a session, and patients would wait as doctors and nurses worked through their allocation. ${ }^{22}$ Such arrangements were predicated upon an economic conception of time as a limited resource, prioritising the efficient flow of medical consultation at the expense of long waits and hurried care for patients. ${ }^{23}$ More importantly, although existing temporal systems divided time into minutes, hours, and clinic sessions, 
they did not track patient attendance or encourage contact with patients who did not appear. Patients might be told to return in three or six months, but before the 1970s providers were seemingly unmotivated or unable to chase patients proactively.

As the frequency and content of patient oversight gained ever greater value, doctors and care teams designed tools to facilitate better tracking of patients. In general practice, the earliest efforts to regulate patient attendance were based upon recall systems, generally in the form of card-index technologies. A Bristol GP, J. M. Wilks, described such a system in a 1973 article for the Journal of the Royal College of General Practitioners (JRCGP) ${ }^{24}$ As for patients with other conditions 'requiring regular surveillance', Wilks kept 'a simple card index system housed in a small box ... on the consulting room desk'. Each patient with diabetes had 'a card tagged with a coloured adhesive label', on which Wilks noted the 'significant' details of the patient's condition. These cards were organised in a month and year pattern, with a 'this month' divider separating those to be seen in the current month from those to be seen in the months coming. Cards in each section were then arranged alphabetically for rapid access during consultation. 'At the end of the month', Wilks went on, 'there should be no cards left in [the] "this month" [section]', but if there were, 'these [patients] can be pursued by letter, telephone or health visitor.' Finally, once each patient had been seen, their card was 'put forward to the month in which it is wished to see the patient next', and the process started again. ${ }^{25}$

For Wilks, one of the advantages of the index system was that it reordered the temporality of care for the patient, making it more closely adhere to the rhythms of the health services. Whilst imposing no specific time for consultation, the system still sought to limit patient choice. Moreover, by substituting the body of the patient for an inscription on card index, Wilks also attempted to ensure that absences would be noted and corrected, thereby actively managing the time between visits. ${ }^{26}$ Yet, alongside closer management of patients, the index tool also inherently regulated the timing of professional labour. The GP's own work was now inevitably tied more closely to bureaucratic 'clock time' and undertaken within a prescribed schedule. ${ }^{27}$ Noting an absence led to telephone calls, with verification rituals undertaken at a specific time at the end of the month. Moreover, as patients returned to the surgery, their reappearance prompted the doctor to undertake review at its scheduled moment. Given the space between visits, and the small number of patients that GPs had on their lists, such effects were 
undoubtedly small. But they were to form part of a broader complex of instruments and actors directed to surveillance that would inevitably manage professional labour.

Over time, tools for oversight developed and doctors and hospital administrators involved new staff in novel patterns of care. For instance, another article, written approximately eight years after Wilks's, described clerical personnel compiling a separate practice register for diabetes patients, and GPs deputing recall to nursing staff. At this surgery in Wales, an attached diabetic nurse oversaw the card index and used appointment details to record who had missed their pre-arranged consultations. The nurse specialist would subsequently send reminders to those patients who had defaulted, and then visit patients who forgot or decided not to attend - a second time. ${ }^{28}$ Although patients could not be forced into attendance, the system closed the mesh of surveillance around a patient, mapping and regulating time between visits. Likewise, whilst the temporal investment in maintaining follow-up had been deputed, both doctor and nurse still followed the rhythms of the new systems in terms of patient review. Similar changes occurred in the equipment itself. In particular, recall became computerised. ${ }^{29}$ Where computers were available, recall packages in general practice had become a standard provision by the late $1980 \mathrm{~s} .{ }^{30}$ Moreover, community care schemes developed systems that proactively assigned patients appointments by letter, and prompted them to attend for laboratory investigations and clinical and optical reviews. ${ }^{31}$ Computerisation, therefore, allowed the temporality of practice to be finely calculated for maximum efficiency and oversight. ${ }^{32}$

To be sure, rhetoric did not always match practice. The functioning of systems depended upon the agents involved acting according to predicted patterns. However, as complex individuals enrolled in various relations with competing demands, doctors and patients varied in their commitment to follow-up. For patients, clinics and their doctors could be remote, producing financial and temporal strains. ${ }^{33}$ Decisions to attend were also based upon complex physical and psychological assessments. One interviewee with diabetes (diagnosed when aged seven in 1976) reflected on how she often had to wait for over an hour and a half for a consultation as a teenager, only to become 'aggrieved' with the discussion. She felt that consultants (then and since) consistently tried to be her 'headmaster or headmistress', which produced problematic dynamics and contributed to a decision to drop out of medical surveillance for four years as a young adult. ${ }^{34}$ 
Staff and instruments could also act in ways counter to the roles envisioned. Zest for recall and proactive care could run dry. As one published review of diabetes management in general practice noted, care deteriorated a year or so after more systematic measures had been implemented: 'fewer patients were seen and the intervals at which they were seen had increased'. Upon examination, 'patient notes suggested this deterioration in care was partly due ... to a decrease in the enthusiasm of the staff running the clinic and recall system. ${ }^{35}$ Even computerisation might fail to compensate for such bureaucratic fatigue. Computerised patient registers and recall systems were costly to construct and run, meaning that projects could run out of money. ${ }^{36}$ Doctors in the late 1980s also bemoaned how temperamental computer systems could be. "Relatively minor typographical errors may "lose" a patient in a computer database', one article warned, and 'interruptions of electrical supply or malfunctions in hardware may lose them all. ${ }^{37}$ Across hospital departments, clerical and medical staff often preferred card index systems, and even maintained them alongside computerised lists. ${ }^{38}$

Teething problems with new instruments, their potential costs, and the resistance of some professional and clerical staff to information technologies were not the only obstacles to NHS computerisation. Policy changes and economic disincentives also exacerbated problems within the hospital sector, resulting in unequal adoption of digital technologies across the health service and generating a considerable legacy for later decades. ${ }^{39}$ Such problems, though, did not prove harmful to the drive for diabetes surveillance. The major professional groups in favour of computerisation and recall systems (senior diabetologists and GPs, service planners and managers) remained steadfast in their faith, and systems were far easier to organise at a local - as opposed to regional or national - level. Advocates did not regard the technical problems they faced as serious enough to disrupt either the underlying clinical need for more refined long-term patient monitoring or the financial savings and managerial control generated by more efficient oversight and distribution of labour. ${ }^{40}$ Instead, with technological change supportive of professional ambitions, these advocates cast disruptions as temporary concerns that could be solved through technical improvements and adjustments in how systems were used. As noted in Chapter 2 , some clinicians and GPs confronting problems in community care schemes continued to see a 'centralised computer prompt' as the basis upon which better surveillance could be secured. ${ }^{41}$ Similarly, those 
practitioners highlighting the importance of enthusiasm saw continuous review and contract incentives as potential solutions. ${ }^{42}$

Though clearly diluted in practice, moves to regulate the temporality of patient review nonetheless had clear managerial effects for practitioners. Structured patient care meant structured medical work. In part, such effects were inherent to the instruments used. As will be seen below, however, such outcomes were also an intended part of plans to combine tools to structure the timing and content of care, and to subject both to review.

Records, protocol, and the management of space, teams, and activity

Alongside recall systems, the development of care protocol and the refining of patient records were also crucial to producing the management of professional labour within systematic and integrated care schemes. However, whereas recall registers regulated the timing of surveillance, records and protocol structured the content of care, as well as rendering the actions of care teams visible.

Along with the growing impetus to disease surveillance, doctors were also prompted to create new tools by the expansion of diabetic care teams. The number and types of healthcare worker involved in diabetes management had risen steadily since the 1920s. Within the most sophisticated and dedicated hospitals, patients before the 1950s might have encountered doctors, almoners, nursing staff, dieticians, and technicians, whilst into the post-war period patients had an increased chance of engaging with obstetricians, chiropodists, ophthalmologists, opticians, and new 'primary care' teams of GPs, practice nurses, health visitors, and district nurses. ${ }^{43}$ This diverse team was also spreading out across space, taking in clinics, wards, GP surgeries, community settings (such as opticians' practices), and patients' homes. New records and care protocol were thus designed to co-ordinate the activity of multiple workers across diverse sites, beyond single institutions.

To understand why GPs and specialists turned to records and protocol to co-ordinate care, however, it is necessary to trace their emergence, and to note that these tools were objects of broader medical interest over the twentieth century. Crucially, clinical records and protocol were both intertwined with broader changes in health services after 1900. As noted in the Introduction, by the 1930s hospitals had 
become complex institutions of mass healthcare. ${ }^{44}$ Specialisation and the increased sophistication of medical technology often meant that multiple practitioners monitored, treated, and interacted with patients (or at least their samples). ${ }^{45}$ Likewise, the emergence of the National Insurance programme introduced notable changes into British general practice after 1913, and state responsibilities for co-ordinated hospital and public health initiatives also expanded dramatically during the early decades of the century. ${ }^{46}$

Such changes transformed administrative and clinical arrangements, especially within hospital settings, and new documentation made further transformations possible. ${ }^{47}$ In terms of records, it was during this period that the personal casebook of the physician and the ward notes of the old hospital transformed into the recognisable case file. ${ }^{48}$ Under new pressures, clinical notes could no longer be the personal preserve of individual doctors, kept stationary in offices or homes. Instead, clinical documents needed to be mobile, following the patient around the growing spaces of the hospital. They also needed to be collectively compiled, providing a record of test results and clinical opinion and offering a means through which the work of various team members could be noted and co-ordinated. Finally, to handle the flow of patients, and facilitate effective analysis and administration, records were subject to drives for internal standardisation and pre-formatting. ${ }^{49}$ Clinical, pathological, and physiological research, just like efficient institutional management, was possible only through the co-ordination of work and the production of focused and comparable records owned by the institution for impersonal analysis. ${ }^{50}$

Equally, as state-funded bodies became involved in cross-institutional research and public health programmes, these agencies used their control of finances and scarce materials to impose new regulations on inter-war medical work. ${ }^{51}$ For instance, in pursuit of controlled 'scientific medicine', the UK Medical Research Council (MRC) developed formally codified protocol for its multi-centre clinical trials. ${ }^{52}$ Similarly, new public health programmes, especially those involving cancer care, attached norms for organisational patterns and information collection to supplies of materials for operating centres. ${ }^{53}$ Indeed, by the midcentury, records and protocol had become objects of overt political interest. Elite practitioners, clinical researchers, service administrators, and Ministry of Health officials all saw the NHS as either an opportunity for research or as an institution in need of significant management. The information that new records might produce was considered of 
great value to service management, as well as to epidemiological and clinical investigations. ${ }^{54}$ Major professional and government bodies introduced centralising pressures - from collective epidemiological research programmes to standard data systems for inpatient analysis that may have encouraged standardisation. ${ }^{55}$ Similarly, during the early 1970s, the Royal College of Physicians of Edinburgh, the Royal College of Surgeons of Glasgow, the RCGP, and the Faculty of Community Medicine formed a joint working party to consider clinical integration. As chronic conditions crossed institutional boundaries the most, the group investigated integration in the care of four common chronic illnesses (dyspepsia, hypertension, stroke, and diabetes). At the heart of their proposals sat the production of clinical protocol to facilitate better specialist-GP interaction. $^{56}$

Such transformations did not always run smoothly. Institutional demands could conflict with cultures of practice in diverse settings. Individualist consultants could ignore protocol, seek control over records, and depute notation to juniors, thus undermining the efforts of reformers. ${ }^{57}$ In the individualistic world of general practice, minor efforts to introduce records as part of National Insurance practice faced strong countervailing winds. ${ }^{58}$ Records only slowly became part of accepted practice, and were completed erratically into the NHS era. ${ }^{59}$ Despite pressures tending towards reform, therefore, a fear of imposing uniform models continued to work against standardisation per se into the 1970s. ${ }^{60}$ The localism of pre-NHS services ensured both a diversity of practice across Britain and a deep attachment to idiosyncratic forms. Standardisation was even thwarted by basic material differences in storage (such as diverse cabinet sizes). ${ }^{61}$

Nonetheless, by the late 1960s, a wide variety of groups within British medicine were interested in co-ordination and data collection. They saw disorganised care as a problem, and envisaged tools like new records and care protocol as possible solutions. Standardising content of care at this point was less important than the fact that disease management required structure. As suggested, diabetes management, along with the care of other chronic conditions, provided an early testing ground for deploying new instruments, and as noted below some practitioners and researchers were interested in diabetes solely for this reason. At the same time, for doctors who turned to records and protocol to co-ordinate care programmes, this pre-existing bureaucratic culture, albeit with local variations, informed subsequent innovation. 
In fact, initial attempts to reform diabetes care records were directly connected to the efforts to standardise hospital medical records noted above, and suffered a similar fate. In 1968, the BDA asked Dr John Bingle, a consultant in York, to review existing clinic documents and to form a panel to consider standardisation. ${ }^{62}$ Bingle maintained communication with the Department of Health and Social Security (DHSS, replacing the Ministry of Health from 1968) and its record reforms committee. ${ }^{63}$ However, although the BDA subcommittee noted that 50 per cent of the hospitals contacted used their own special charts for routine follow-up (all with similar layout), the proposed standard, composite, form (see Fig. 3.1) did not survive the transition out of committee. Bingle noted that clinic 'requirements ... varied so much' that a

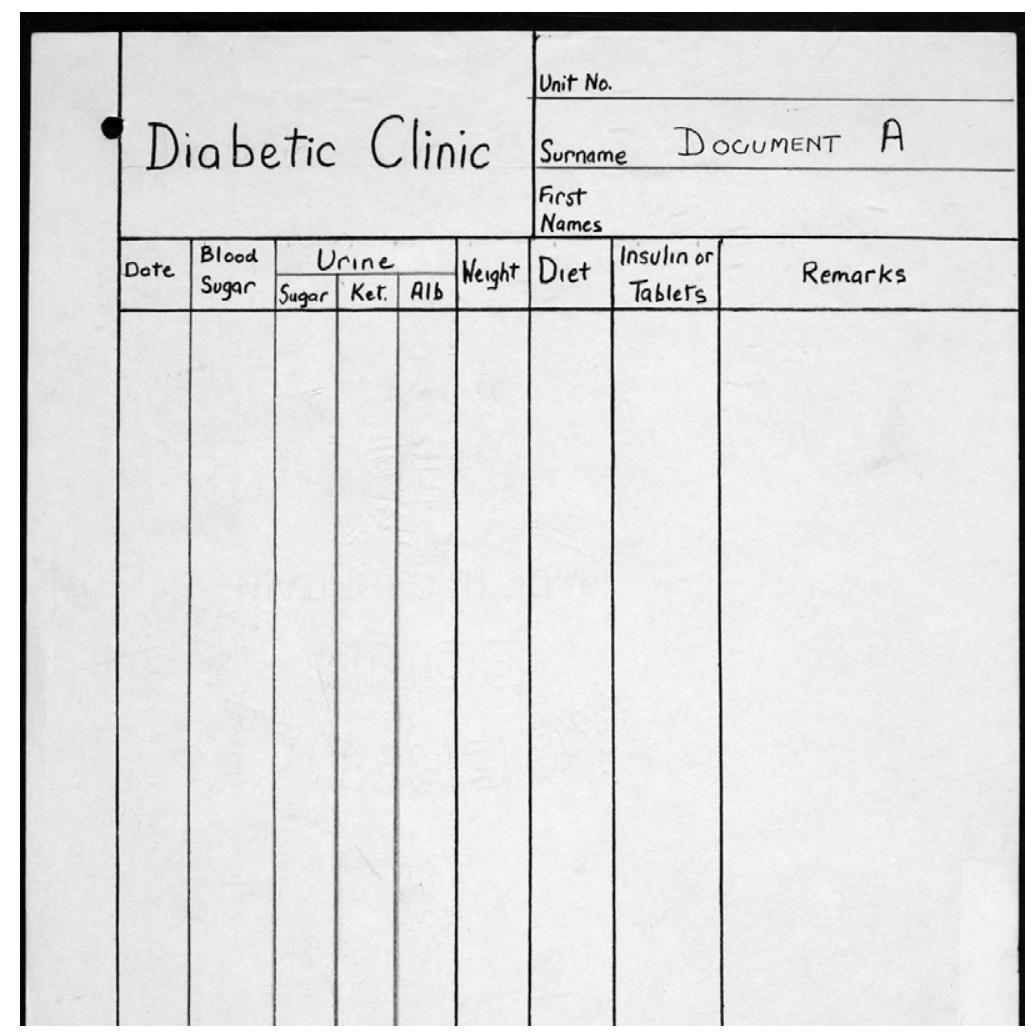

Figure 3.1 Standard outpatient clinic review form, c.1968 
single form could not suit all purposes, and that 'there is considerable apathy over documentation, each clinic having at some time designed its forms and being somewhat unwilling to change to a new type without a very good reason. ${ }^{64}$

The record itself, however, was noteworthy for the regulatory and clinical values it embodied. As with earlier innovations in records for inpatient diabetes care, the pre-formatting of the record could be seen as an attempt to influence practice. ${ }^{65}$ Lined sections of the form may have restricted the data gathered and passed on, thus ordering the work to be performed. ${ }^{66}$ Moreover, the subcommittee had to reflect on what they saw as the most important data to be collected, and members clearly prioritised biochemical markers (blood glucose, glycosuria, ketonuria, and albuminuria), as well as weight and treatment information. ${ }^{67}$ They appeared to agree, therefore, that good care involved collecting physiological and clinical information at the very least. At this stage, this control-through-communication may have been somewhat limited, as the record still allowed a large unstructured column for qualitative remarks. ${ }^{68}$ Unlike later forms, moreover, this record would have formed part of a range of outpatient observation documentation, with less structured forms perhaps providing additional data.

Although clinic doctors of the 1970s resisted efforts to standardise care nationally, these same practitioners, along with GPs, slowly introduced bureaucratic tools that managed practice at the grassroots level. As with recall, renewed concerns with patient surveillance motivated doctors to introduce new instruments to structure monitoring, often after service reviews revealed deficiencies. For instance, one early and influential study conducted by a trainee GP remarked how the notes in one general practice suggested that 'biochemical investigations relied completely on the patients' own recordings, with no supervised check being carried out. ${ }^{69}$ Some tests were erratically performed. Blood sugar examinations, for example, were seemingly carried out inequitably 'recorded more often for diet and diet plus oral hypoglycaemic controlled diabetics than in insulin-controlled cases' - and the general conclusion was that 'one could not rely on the last entry in the notes to indicate the biochemical control of the patient's diabetes'. Just as importantly, the recorded information 'was often available only after prolonged searches through sheaves of results and letters from clinics. ${ }^{70}$ The reviewer thus noted three interlinked problems, all of which were a concern given the importance of surveillance and the potential deputation of further responsibility to GPs: key parts of medical work were 
not being undertaken regularly; when work was performed, doctors had not recorded it; and, when recorded, the results of this work had not been noted clearly.

Within this single site, the reviewer saw the solution to inconsistencies of surveillance in three parts. Firstly, they recommended designing an effective recall system. Secondly, they proposed more systematically dividing the responsibilities of practice nurse (biochemical tests, weighing, dietary advice) and GP (clinical interview, screening for complications, and record completion). Finally, the author stressed the need to create 'an effective follow-up chart' (Fig. 3.2)..$^{71}$

The new record card was crucial. The reviewer designed it to solve multiple problems of poor surveillance and recording. The survey section, for example, included a 'simple tick system', enabling easy completion at the front of the form. ${ }^{72}$ Through design, the effort required to complete the necessary notes could be reduced and dismantled as an obstacle. Furthermore, this formatting was also intended to structure the practitioner's surveillance of the patient, and correct the problem of work being neglected. On one level, whilst retaining flexibility in its ongoing clinical sections, the record used visibility to encourage adherence, providing clearly marked spaces to note test results, problems, and a 'plan' of action. Of course, notes did not need to be made, and certain actions could easily be forgotten or not undertaken. In such instances, however, the record's spatial zoning would make absences clear to GPs, and would allow them an instant review on a yearly basis of [the] routine care and advice offered. Finally, going beyond visibility, the record itself was created to actively prompt work when in use. The author, for instance, noted that there would be a 'built in reminder' for GPs to screen for complications. The record's minute detailing of the checks to be undertaken within the annual review provided this cue, indicating to the user what tests should be performed as it was being completed. ${ }^{73}$ An aide-memoire to previous work was thus transformed into a prompt when the record was in action.

Finally, it is worth noting how the reviewer designed the record to facilitate new kinds of teamwork. Responding to calls for GPs to adopt mini-clinic sessions for efficiency purposes, the author noted that 'with the co-operation of receptionist, practice nurse and doctor' records could be completed adequately, and 'dual appointments to see nurse and doctor could be fitted into a normal surgery session. ${ }^{74}$ The reformulated record, therefore, was intended to be an active part of a broader technology of surveillance, one that facilitated and prompted action 


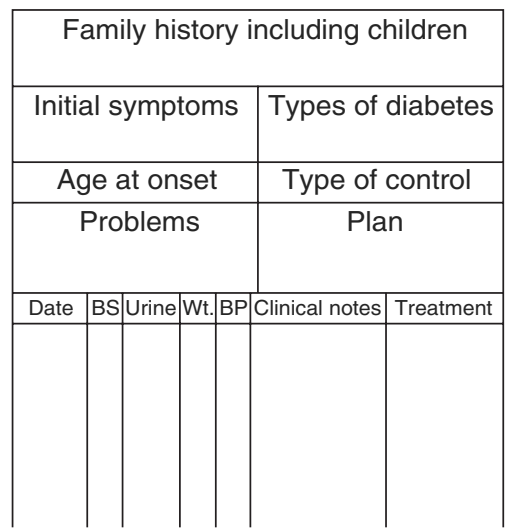

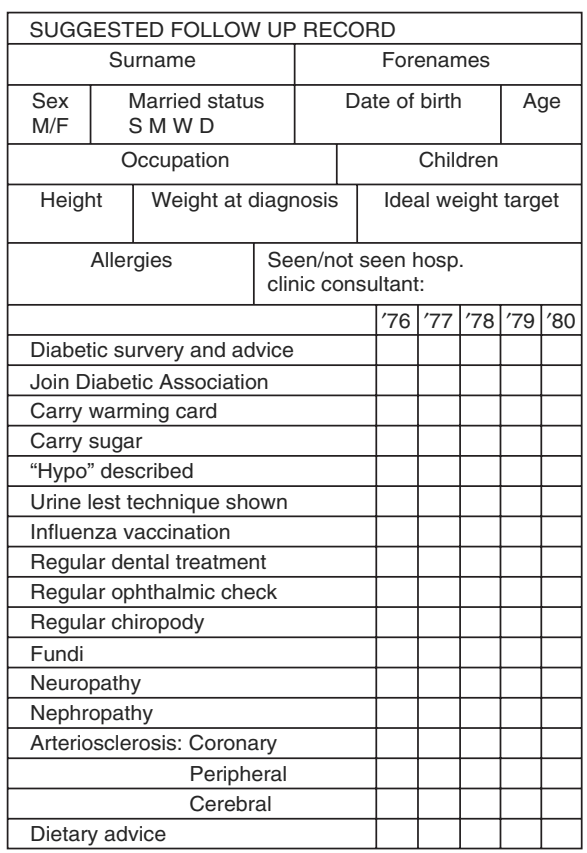

Source: A. P. Kratky, 'An audit of the care of diabetics in one general practice', JRCGP, 27:182 (1977), 536-43.

Figure 3.2 Components of a new record card for GP diabetes care, c.1977 
and enabled new forms of working and divisions of labour. The record thus played a 'constitutive role' in diabetes care, both inherently and by design. ${ }^{75}$

Over time, more GPs came to adopt such special records. Careful zoning, preformatted priorities, and even 'stamps' for annual reviews became core features of new documents. The RCGP promoted records along these lines as part of its Quality Initiative, including existing models of diabetes records in its 'clinical information pack' for the condition. ${ }^{76}$ Indeed, the pack was the second that the College produced, reflecting the importance of diabetes as a gateway to 'the development of ... expertise [in] preventive aspects of medical care' and to issues of 'practice organisation and management. ${ }^{77}$

That early impetus for reform in general practice appeared to come from more junior members of the profession probably indicates that a generational shift contributed to the spread of new approaches to clinical activity. Recent involvement in forms of hospital practice and pioneering vocational GP training may have exposed junior practitioners to cutting-edge concepts of good care centred on surveillance and bureaucracy. ${ }^{78}$ Equally, changes in general practice's material and intellectual foundations provided fertile ground for an emphasis on records at this point. Crucially, group practice had slowly become the new norm in primary care, driven by professional interest in peer exchange and financial and organisational imperatives of sharing costs and workload. The result was to undercut 'continuity of care', and doctors could no longer rely on a personal relationship to know their patient's histories. ${ }^{79}$ GPs therefore needed records to provide an aide-memoire, as well as to encourage unfamiliar staff to adopt common practices. ${ }^{80}$ In a similar vein, the growth of the 'primary care team' within general practice was also influential here, with records facilitated by (and facilitating) the incorporation of specialist clerical staff, practice nurses, and attached health visitors, chiropodists, dieticians, and community nurses following the GP contract of 1965 and NHS reforms of $1974 .{ }^{81}$

A more challenging version of this growing care team, however, appeared in integrated care programmes. Unlike systematic care within general practice, surveillance and therapeutic titration in these schemes occurred in multiple sites (across hospitals, general practice surgeries, and patients' homes), posing several possible problems. Firstly, if schemes were left uncoordinated, patients might fall through gaps in provision, or receive different standards of care based on institutional 
geography. Secondly, without a clear division of responsibility between practitioners, the system might produce an inefficient repetition of work. And finally, even if lines of communication were established, information could be lost or unintelligible without a formal language shared across services and a changing roster of staff. Within such circumstances, doctors and other 'stakeholders' created tools to clearly allocate, communicate, and review work undertaken across different segments of integrated services.

Records were once again important. In perhaps the most fully realised shared care scheme of all - that in Poole - a working party of GPs and a consultant produced a new form of diabetes record (in collaboration with the local hospital management committee and Hoescht Pharmaceuticals): a 'community care co-operation booklet' that travelled with the patient between practitioners and sites of care (see Fig. 3.3) ${ }^{82}$

The premise of a mobile record itself was not entirely novel. As the designers of the Poole system noted, GPs and hospital practitioners had shared cards for other types of patient, primarily for 'chronic schizophrenics' and antenatal care. ${ }^{83}$ However, the community care booklet served multiple functions. It contained advice on diet, 'foot care, illness, insulin reactions, driving, and general instructions about attending the laboratory and general practitioner'. Through its qualitative elements, therefore, it sought to influence the work of its users. Its clinical section also implemented many of the changes in record forms already noted. It contained ruled sections for dates of observation, test results, notes on treatment, and a 'check list to indicate when the patient was last assessed for diseases of the eyes, nervous systems, cardiovascular system, and the feet. ${ }^{84}$

Perhaps more than when records remained in a single institution, this zoning of the form was crucial to the management of the scheme and its spatially dispersed practitioners. Such techniques, for instance, reduced the possibility of idiosyncratic shorthand, regulating information exchange so that staff across different settings could interpret notes without misunderstanding. Moreover, by clearly boxing specific data, the record could prevent 'wasteful duplication of effort' and allow errors to be checked and corrected. ${ }^{85}$ Indeed, the Poole clinical lead hoped that computerisation would provide a 'method of assessing the followup and control of patients not regularly seen in the clinic. ${ }^{86}$ Although it was potentially a hospital plan to manage GPs, no punitive measures could be devised in a programme dependent upon co-operation. ${ }^{87}$ 


\section{Clinical record}

\begin{tabular}{|c|c|c|c|c|c|c|c|c|c|c|c|c|c|c|c|c|}
\hline \multirow{2}{*}{ Date } & \multirow{2}{*}{ WT. } & \multicolumn{2}{|c|}{ Blood Test } & \multicolumn{3}{|c|}{ Urine } & \multicolumn{4}{|c|}{ Check list } & Diet & \multicolumn{3}{|c|}{ Insulin } & \multirow{2}{*}{ Oral therapy } & \multirow{2}{*}{$\begin{array}{l}\text { NEXT } \\
\text { VISIT }\end{array}$} \\
\hline & & I.B.S. & $\mathrm{HbA}_{1 \mathrm{C}}$ & $S$ & $P$ & Ac. & Eyes & N.S. & C.V.S. & Feet & G.Cho. & Type & Units & $\begin{array}{l}\mathrm{am} / \\
\mathrm{pm} /\end{array}$ & & \\
\hline & & & & & & & & & & & & & & & & \\
\hline & & & & & & & & & & & & & & & & \\
\hline & & & & & & & & & & & & & & & & \\
\hline & & & & & & & & & & & & & & & & \\
\hline & & & & & & & & & & & & & & & & \\
\hline & & & & & & & & & & & & & & & & \\
\hline & & & & & & & & & & & & & & & & \\
\hline & & & & & & & & & & & & & & & & \\
\hline & & & & & & & & & & & & & & & & \\
\hline & & & & & & & & & & & & & & & & \\
\hline & & & & & & & & & & & & & & & & \\
\hline & & & & & & & & & & & & & & & & \\
\hline & & & & & & & & & & & & & & & & \\
\hline & & & & & & & & & & & & & & & & \\
\hline & & & & & & & & & & & & & & & & \\
\hline & & & & & & & & & & & & & & & & \\
\hline
\end{tabular}

Figure 3.3 The community care co-operation booklet, c.1980s 
The designers simply hoped that the record would increase visibility of errors for care teams, with practice review forming the basis for changed behaviour.

During the 1970s and 1980s, hospital hopes for computerised surveillance of professionals were seemingly unrealised. Constrained NHS funding underpinned technical difficulties. ${ }^{88}$ Furthermore, doctors using similar records complained that such tools were - in themselves - unable to ensure that professionals always performed 'relevant' disease management. ${ }^{89}$ Such problems did not prove fatal for the Poole scheme, or for the record form itself. Hoescht Pharmaceuticals published the booklet as a commercially available product, and GPs and other community care schemes adopted and adapted it. ${ }^{90}$ Even the RCGP indirectly recommended it. ${ }^{91}$ In this sense, the adoption of the record mirrored the spread of similar forms discussed above.

It was in this form of integrated, structured care that diabetes management appeared as something of a model for other chronic conditions. The interest of the Royal Colleges has already been highlighted, with diabetes appearing as an exemplar of proactive and preventive medicine, existing at the forefront of cross-institutional experiments in integrated chronic disease care. Yet the influence of diabetes care on practices in other conditions did not always derive from success. One article, for instance, detailed how the authors had heard about 'an experiment in shared hospital-GP care of diabetes' which reported 'only limited success, despite careful planning. They were thus inspired to detail their own experience in the management of hypertension, to address fears that 'shared care of hypertensives would fare no better and that the portable record would be lost, forgotten, badly completed, or illegible. ${ }^{92}$ Despite problems, the use of mobile records and shared care systems nonetheless situated diabetes care at the vanguard of trials in service management, and formed part of a broader discourse around chronic disease and service integration.

However, the power of the record to structure care was truly realised only in combination with care protocols. As noted, protocols for medical practice had numerous origins, and from these sites they seeped into clinical practice after the Second World War. In Britain, the growth of clinical research within the NHS helped to familiarise doctors with using and designing 'protocols', positioning them as a sensible option for co-ordinating action across multiple sites. ${ }^{93}$ From the 1970s onwards, several integrated diabetes care programmes used formal protocols to facilitate effective functioning of diverse moving parts. According to 
early debates, initial agreements may have included 'the circumstances in which patients could or should be kept at home for treatment by the general practitioner, the pattern of investigation of such cases, and the criteria for referral to hospital. ${ }^{94}$ The Poole scheme even supplemented such agreements with extra documentation advising patient and GP about follow-up and 'the standard of control expected.95

Other schemes went further as time passed, tightening the regulatory aims of their protocol. The Ipswich community care scheme, for instance, selected patients for hospital and general practice care using clear criteria, including strict biochemical thresholds of control, age, and various risk and clinical factors. Formal procedures were established for referral and discharge. Furthermore, although GPs were offered freedom of organisation, they agreed to meet 'standards of care, follow-up, and recording', including (1) at least yearly review of 'wellcontrolled' patients, and either more frequent observation or referral to hospital if control fell outside of agreed parameters; (2) measurement and recording of a minimum agreed set of base surveillance metrics (blood glucose, glycosylated haemoglobin (HbAlc), urine tests, weight); and (3) at least annual assessment of visual acuity, foot condition, and blood pressure, and performance of fundoscopy. ${ }^{96}$

As will be discussed in Chapter 5, by the end of the 1980s, professional organisations were producing model protocols with more finely tuned arrangements, and even GPs outside integrated programmes were promoting their use. ${ }^{97}$ The RCGP protocol, for instance, contained a cartographic attachment: the 'diabetes flow-chart', an A1-sized poster which was most likely to be hung on a practitioner's wall for easy consultation. ${ }^{98}$ Like other types of mapping, the flow-chart was an exercise in abstraction and legibility. ${ }^{99}$ It disaggregated diagnosis and management into certain 'pathways' to be taken if specific conditions were met or not. In so doing, the map embodied an idealised route to care, one that presented it as a simple process of decision-making devoid of contextual factors (about patients and institutions). ${ }^{100}$ Crucially, through this decontextualisation and direction, the flow-chart represented a tool to guide patient management, visualising the 'correct' steps to take and highlighting deviations by their absence.

Interestingly, the map also presented a very specific view of care. It contained no 'patient-centred' indicators, such as whether patients were happy with treatment plans. Instead, conditions determining action were clinical and biochemical. It may be that discussions of patient experiences and feelings were informal, the unrecorded product of 
exchange between practitioner and patient. And this is hinted at in certain protocol that contained points for discussing 'wellbeing. ${ }^{101}$ However, their relative marginality within tools for surveillance is nonetheless notable given how designers used these instruments to prioritise key information and tasks, and to manage professional labour.

\section{Audit, investigatory medicine, and the consolidation of professional management}

In each of the tools considered so far, we can trace the emergence and reproduction of a consensus about 'good' diabetes care. Unlike in later years, neither hospital doctors nor GPs defined quality care in terms of outcomes or targets during the 1970s and 1980s. Instead, their records and protocol focused on care process and the same set of physiological markers, checks for complications, and clinically important data on treatment. ${ }^{102}$ Moreover, pioneers of GP-based and integrated care all saw a combination of tools as essential to securing good care, coordinating the efforts of care teams, and ensuring regularity and content of oversight. These innovators even slowly articulated the inherently managerial ethos of these new technologies, noting how they prompted activity and recorded performance.

In terms of diabetes, the existence of a professional 'good sense' is perhaps unsurprising. The very creation of the instruments in question forced designers to clarify what they saw as the elements of good care, and the BDA provided a network through which medical professionals could discuss their ideas. As Figure 3.2 above indicates, the organisation exercised considerable influence by the 1970s, and elite bodies like the Royal Colleges also connected interested practitioners and promoted model records and protocol imbued with the emergent consensus. ${ }^{103}$

This articulation of good care, and its connection with regulatory instruments, marked something of a shift in understandings of quality in medicine. During the first half of the twentieth century, doctors (and society more broadly) held that proficiency in medical practice could be guaranteed through effective training and experience. Of course, as noted in this and earlier chapters, professional action would be influenced by any number of other factors. Doctors working in institutions and hospital 'firms' might become socialised into certain approaches, and were constrained by the facilities they had available. Likewise, medical handbooks and journals could provide up-to-date information on trusted or innovative procedures, whilst hospitals might deploy their 
own documentary aids to help practitioners to tailor observation or therapeutic practices. ${ }^{104}$ However, 'the right of private judgement' and clinical individualism were highly prized, and professional action was rarely subject to codified criteria of proper practice, temporal regulation, or recording. ${ }^{105}$

Taken together, therefore, recall systems, specialist records, and care protocol added new local structure to professional action. As discussed in Chapter 5, this structure emerged at a time when professional, public, and political actors had grown concerned about the competence of medical professionals. Professional self-reflection emerged from a cacophony of sources, from media scandals about insensitive care in long-stay institutions to growing critiques of welfare professionals as self-interested bureaucrats (rather than altruistic servants), and from academic studies of variations in care to the rising status of trials as technologies for determining 'best practice'. All contributed to a growing sense that quality care could be secured only through formal regulatory devices.

Devices to structure medical practice could become a 'managerial' technology in a more formal sense, though, only with observation and review. The power of norms derived, in part, from the possibility that adherence, omission, or deviation would be visible to self and others. ${ }^{106}$ Thus it was only when recall, records, and protocol were connected to medical audit that their capacity to reshape and manage professional action was fully realised. As noted in the coming chapters, different visions of professionalism and management emerged in relation to protocol and audit. At least within the local systems discussed here, management did not involve formal discipline for deviations, or review being explicitly tied to resource allocation and lay intervention. Rather, doctors saw audit and related tools as educational and as useful for reforming their own practices and systems. ${ }^{107}$

This educational element was perhaps most clearly articulated in the concept of the 'audit cycle.' 'Assessment', noted one influential audit of diabetes care, 'will show in practice if aims are met', quantifying the extent to which this was the case. However, it continued, 'there must be a feedback from "assessment" to "aims", modifying the aims and therefore altering the pattern of care offered'. In this sense, audit was taken to 'represent a continuous process of thought', with 'continuing changes in aims ... measured in terms of changing levels of care (assessment). ${ }^{108}$ Here, protocol could provide a set of standards against which care could be assessed, whilst records and recall systems provided the 
traces of care upon which analysis would act. ${ }^{109}$ Conversely, it was also the case that audit enclosed diabetes care (and medical care more broadly) in a cycle of self-review and management. Without observation, structures could be ineffective and visible actors could deviate. Discipline may have been productive of new forms of working, but it required monitoring. ${ }^{110}$

Audit was a novel practice in British medicine before the 1970s. International influences were important to helping it develop, with American experiences filtering into British discourse. ${ }^{111}$ Domestically, doctors themselves were not separated from the society and culture that was making demands for professional regulation. They shared its modes of thought and had knowledge of developments in other professions. ${ }^{112}$ Indeed, audit was initially a technology of financial accounting, and British GPs and hospital clinicians had long had connections with private business. ${ }^{113}$ In earlier decades, for instance, they had conducted (and been participants in) time-and-motion studies to assess organisational efficiency, hospitals in the late 1960s had drafted in management consultants to improve their administration, and some GPs had even sought to explore general practice in terms of management theory. ${ }^{114}$

Developments within British medical discourse and practice were also of importance. Psychologically oriented theories emerging in the late 1950s emphasised professional fallibility and reflexivity, whilst the NHS's close connection with research saw major figures in British clinical and health services research promote techniques of review as central to efficiency and efficacy. ${ }^{115}$ In fact, some doctors saw diabetes as a site for developing audit itself. Chronic disease care developed a rich culture of inscriptions and registers. The supposedly document-heavy nature of diabetes management, therefore, made it a promising target for promoting audit, as did its quantified diagnostic criteria. As one GP put it when justifying their choice of subject for developing audit, diabetes was 'more readily defined than ... many of the chronic diseases', and its patients were 'easier to identify.'

Doctors initially audited their diabetes management arrangements, then, as an investigative practice, establishing a co-constructive relationship between talk of standards, structured care, and management. As noted above, early investigators were sometimes concerned that expanding the care team might lower standards, or they sought to demonstrate that improvements in care were possible. As audit occasionally preceded new arrangements, auditors established the very measurements through which it became possible to discuss 'standards' of care. 
As one study, cited above, put it, 'one has to set standards against which to measure. ${ }^{117}$ In this instance, the reviewer combined social, clinical, and biochemical factors to classify diabetic care as 'good', 'moderate', or 'poor'. Ultimately, the author found effective audit difficult to conduct, and thus problematised what they saw as deficiencies in record-keeping, oversight, and management documentation. In this case, potential obstacles to audit became legitimation for reforming care processes and structures, and for further audit itself. ${ }^{118}$ It was a co-constructed pattern followed elsewhere. ${ }^{119}$

Auditing also had a close relationship with health services research that assessed the functioning of novel schemes for diabetes management. Though encompassing process measures, reviews of services also variously added intermediate outcome measures (such as $\mathrm{HbAlc}$ results) that may have formed part of local protocol standards. ${ }^{120}$ Whilst such reviews were not always labelled 'auditing', participants expressly framed their investigations in relation to concerns about reformulated care responsibilities. ${ }^{121}$ Reviews of novel programmes appeared within a few years of new arrangements being established, and were considered necessary 'to assess the efficacy' of schemes and even to provide 'baseline' information for ongoing evaluation. ${ }^{122}$

It was through this longitudinal assessment that investigative forms of review later became routine practice, providing a 'feedback loop' into clinical medicine. ${ }^{123}$ Towards the end of the 1980 s, assessments became more formally framed as audits, whilst incorporating measures pioneered in health services research and local protocol. ${ }^{124}$ Although published, such work provided ongoing evaluation, as opposed to consisting of retrospective analyses that promoted or critiqued alternative forms of organisation. New guides to audit stressed the importance of this distinction, arguing that older forms of evaluation were helpful in 'focusing the subject', but were 'concerned with the success or failure of some aspects of diabetes care[,] rather than providing a continuing assessment of management of this chronic disease from which further improvements can be made. ${ }^{125}$ Using the tools developed within this research, this guide now promoted embedding review as part of new service organisation.

\section{Conclusion: did structured care produce a new order?}

The implementation of audit completed a major shift that had taken place in diabetes care after the 1960s. By the 1980s, discussions of 'good 
diabetes care' persistently invoked structured care. Systematic organisation, recording, and review provided signs of good practice, and practitioners believed them to be crucial to guaranteeing effective patient management. In part, hospital clinicians and GPs (supported by specialist nurses, Community Physicians, and other interested parties) had mobilised recall systems, specialist records, and care protocol to improve patient oversight. However, the combination of these instruments, especially with audit, inherently ordered medical labour. As criticism of professional competence spread across British society, designers of new systems intensified the regulatory aspects of the new instruments. When combined, these tools formed local organisational technologies constructed in the name of 'quality', just as clinical trials formed an organisational technology in pursuit of 'truth. ${ }^{126}$ Such technologies were reproduced throughout Britain, beginning in areas with motivated and experienced practitioners, before moving slowly into the 'periphery'.

The question that now needs to be answered, therefore, is: what effects - if any - did this new technology have on practice?

A traditional response might be framed in terms of 'success'. Did care improve? Practitioners assessing new approaches varied considerably on this point. Organisers of the Wolverhampton 'mini-clinic' scheme, for example, expressed consistently positive evaluations, in terms of both process and outcomes. ${ }^{127} \mathrm{New}$ tools apparently facilitated increased oversight, as well as successful co-operation between participants. Likewise, organisers of a community scheme in Norwich noted improvements in recording practices and follow-up, even though considerable omissions remained. ${ }^{128}$ By contrast, reviewers of the nearby Ipswich scheme criticised GPs for continuously poor testing, follow-up, and record completion, despite agreed policies. ${ }^{129}$ And in Sheffield, worse results followed. ${ }^{130}$

Yet posing this question - or at least answering it in terms of audit results - is an effect of the changes being discussed. Instead, a more pertinent route of enquiry might be to ask whether the new combinations of concepts, instruments, and actors produced a new, structured order in diabetes care. ${ }^{131}$ Did the developments traced succeed in making new ways of working possible, and in introducing new forms of professional management on a local level?

Answers can be drawn only indirectly. For instance, discussions of deputation, routine, and repetition in medical texts indicate how some doctors reacted against the managerial effects of new technologies. 
During the 1980s, for example, guides to establishing general practice diabetes care argued that 'nurses are generally better trained in the repetitive tasks of measuring height, blood pressure, etc, than doctors' and 'are more reliable at recording them'. Consequently, 'the doctor should do more complex clinical tests, assess the basic information collected by the nurse and decide on necessary changes in management', as well as organise referral. ${ }^{132}$ The tasks deputed to nurses were those most heavily subject to new managerial pressures, whilst clinical assessment was less able to be reviewed critically. Such language mirrored that applied by consultants and specialists earlier in the century. As seen in Chapter 2, professionals responded to patient load by routinising care for certain patients, and clinicians tried to depute this work to either GPs or non-medical staff (such as dieticians). ${ }^{133}$ By the later 1980s, the routinising pressures of protocol and records saw tasks deputed again, from GPs to nurses. GPs in certain schemes became mini-consultants, with specialist and practice nurses running their own clinics. ${ }^{134}$ To be sure, deputation of work could indicate that practitioners had found a way to negotiate the management of their activities, both in hospital and in general practice. Deputation may also have been interpreted as a positive sign by doctors, freeing them for more complex parts of consultation, such as discussing wellbeing issues. Regardless, deputation provides a sign that managerial pressures had successfully altered care to the extent that negotiations were necessary.

Yet perhaps it is this view of care as constantly changing, but never quite fully changed, that is most productive. One study of audit cycles in a general practice provides insight here. Through the practice's first audit in 1983, the partners noted that there had been 'no structured diabetic care in the practice and the process of care was haphazard'. Accordingly, the practice subsequently developed a patient register and 'a protocol for the care of ... diabetic patients ... which included a routine format for history taking and regular examination, suggested biochemical aims of treatment, and gave aims for education and support'. In 1990, the partners undertook a second audit. They noted that 'the protocol developed after the 1983 audit changed and organised the process of care in the practice', but the new audit was designed to 'examin[e] whether the conclusions of the previous audit had been implemented and whether the defined standards for the process of care and outcome of care ... had all been achieved. ${ }^{135}$ To this, they concluded 'no'. Further work was required, and new discussions were held about 'changes in the protocol and its monitoring to improve future 
performance. ${ }^{136}$ In other words, the study revealed that diabetes care had not been completely reformulated. Standards were missed, and some processes remained unfulfilled. Yet standards and preformatted practices of care did now exist, and deviations were being captured, subject to review, and structures subsequently reformulated. Substantial change had been implemented, and the combination of new tools subjected local practice to management, but no endpoint had been reached.

That this audit was another of general practice raises the question of whether managerialised care was an imposition of specialists upon GPs. Contemporary sociological investigation of audit indicated that traditional hierarchies may have protected consultants from review in some institutions. ${ }^{137}$ Furthermore, as hospital clinics had been considered the 'orthodox' site for diabetes care, it was general practice that had come under suspicion with reorganisation.

The role of professional elites in designing and promoting tools of national clinical governance will be discussed in the coming chapters. At the local level, assumptions that professional management was a hospital invention would overlook the complex dynamics at play. Regardless of their supposedly superior status, hospital practitioners were not the formal managers of GP labour. Consultants could not direct GPs - or punish them for deviations - in the same way as superiors' within a traditional bureaucratic chain. Integrated diabetes care depended upon voluntary arrangements, and less motivated GPs or hostile consultants tended to clash with agreed arrangements. One participant in the Sheffield scheme acknowledged the importance of such mutual investment, noting how consultants 'didn't think general practitioners could look after people with diabetes at all. ${ }^{138}$ As a result, something akin to 'turf wars' broke out before mobile specialist nurses eventually rescued the scheme. ${ }^{139}$ By contrast, elsewhere GPs were part of committees for creating protocols and designing records, as well as undertaking audit. In fact, GPs were themselves pioneers in promoting such tools, and individual practitioners frequently reviewed and reorganised their own practice independently of any official community scheme.

Professional management in diabetes care at a local level, therefore, was generally constructed out of a shared sense of what 'good practice' meant, and relied upon charismatic pioneers and dedicated staff to work. ${ }^{140}$ Debate existed about the importance of specific markers or how to measure potentially immeasurable elements, such as social 
and psychological wellbeing. ${ }^{141}$ Nonetheless, over the decades following the 1960s many figures involved in diabetes care converged on an agreed set of quantifiable indicators, and placed great emphasis on the organisation and structure of care to secure quality. Designers of systematic schemes often adapted tools from existing instruments, reflecting - as did their drive to integrate care - their socialisation within a bureaucratic medical culture The reorganisation of the NHS in 1974 may even have supported such developments, bringing professionals with epidemiological and evaluative expertise closer to clinical institutions. Drawing on their abstractive, 'administrative way of knowing', such individuals were important facilitators and evaluators of new schemes. ${ }^{142}$ When instruments for regulating time and co-ordinating activity were thus combined in coherent schemes with practices of review, the resulting technology subjected professional work to management, producing bureaucratising effects of codification, division, re-integration, and oversight. These effects were not totalising, but were nonetheless substantive, and diabetes provided a leading edge for their implementation.

In the chapters that follow, we will explore how support for managed care developed as a guarantor of quality. These chapters open up a broader vista on political and cultural context as well as international developments. This changing lens is necessary if we are to understand how local developments transformed into (and interacted with) national, and international, programmes of clinical governance. Before this latter history can be traced, however, it will be necessary to make a detour via diabetic retinopathy. Through studying the specific policy debates around retinopathy, we will see how the developments focused upon so far manifested in a single issue. We will also chart how diabetes returned to the policy agenda after the Ministry of Health issued its standards for diabetic clinics in 1953. This exploration will highlight the main players involved in shaping policy and how they interacted with one another, thereby providing a crucial map for historicising developments during the end of the twentieth century.

\section{Notes}

1 L. Winner, 'Do artifacts have politics?', Daedalus, 109:1 (1980), 121-36.

2 M. Foucault, Discipline and Punish: The Birth of the Prison, trans. A. Sheridan (London: Penguin, 1991 [1975]). 
3 S. Timmermanns and M. Berg, The Gold Standard: The Challenge of Evidence-Based Medicine and Standardization in Health Care (Philadelphia: Temple University Press, 2003).

4 H. Marks, The Progress of the Experiment: Science and Therapeutic Reform in the United States, 1900-1990 (Cambridge: Cambridge University Press, 1997). See also discussions of 'networks' and 'systems' in W. E. Bijker, T. P. Hughes, and T. Pinch (eds.), The Social Construction of Technological Systems: New Directions in the Sociology and History of Technology, Anniversary Edition (Cambridge, MA: MIT Press, 2012 [1987]), pp. 11-44, or of 'platforms' in P. Keating and A. Cambrosio, 'Cancer clinical trials: the emergence and development of a new style of practice', Bulletin of the History of Medicine, 81:1 (2007), 197-223, esp. pp. 198-9. Each term belongs to a specific theoretical school. My use of 'technology' derives from my interests as a social historian of medicine, and is to be differentiated from network and platform approaches.

5 M. Weber, The Theory of Social and Economic Organisation, trans. A. M. Henderson and T. Parsons, ed. T. Parsons (New York: Free Press, 1966 [1947]).

6 R. D. Hill, Diabetes Health Care: A Guide to the Provision of Health Care Services (London: Chapman and Hall, 1987), p. 21.

7 H. Valier and R. Bivins, 'Organization, ethnicity and the British National Health Service', in J. Stanton (ed.), Innovations in Health and Medicine: Diffusion and Resistance in the Twentieth Century (London: Routledge, 2002), pp. 37-64; C. Sinding, 'Flexible norms? From patients' values to physicians' standards', in W. Ernst (ed.), Histories of the Normal and the Abnormal: Social and Cultural Histories of Norms and Normativity (London: Routledge, 2006), pp. 225-44.

8 A. Mold, Making the Patient-Consumer: Patient Organisations and Health Consumerism in Britain (Manchester: Manchester University Press, 2015).

9 A. M. Brandt and M. Gardner, 'The golden age of medicine?', in R. Cooter and J. Pickstone (eds.), Companion to Medicine in the Twentieth Century (Abingdon: Routledge, 2003), pp. 29-34. See Chapter 5 below.

10 W. R. Arney and B. J. Bergen, Medicine and the Management of Living: Taming the Last Great Beast (Chicago: University of Chicago Press, 1985).

11 Sinding, 'Flexible norms?', p. 237; R. Tattersall, Diabetes: The Biography (Oxford: Oxford University Press, 2009), pp. 162-4.

12 Tattersall, Diabetes, p. 152.

13 J. T. Ireland, W. S. T. Thomson, and J. Williamson, Diabetes Today: A Handbook for the Clinical Team (Aylesbury: HM+M, 1980).

14 Hill, Diabetes Health Care, p. 21.

15 M. O. Aveline, D. K. McCulloch, and R. B. Tattersall, 'The practice of group psychotherapy with adult insulin-dependent diabetics', Diabetic Medicine, 2:3 (1985), 275-82. 
16 Hill, Diabetes Health Care, p. 91.

17 Ibid., p. 37.

18 Ibid., p. 91.

19 R. D. Lawrence, The Diabetic Life: Its Control by Diet and Insulin. A Concise Practical Manual for Practitioners and Patients, 6th edition (London: J. \& A. Churchill, 1931); J. Malins, Clinical Diabetes Mellitus (London: Eyre \& Spottiswood, 1968).

20 H. P. Himsworth, 'Management of diabetes mellitus, part II', BMJ, 2:3942 (1936), 188.

21 'Organization of out-patient departments', BMJ, S.1:2665 (1956), 55.

22 G. Forsyth and R. Logan, Gateway or Dividing Line? A Study of Hospital Outpatients in the 1960s (Oxford: Oxford University Press for the Nuffield Provincial Hospitals Trust, 1968), p. 221; interview with J. Harkness conducted by the author. Harkness trained and worked as a manager in the NHS from the early 1970s to the 1990s.

23 D. Armstrong, 'Space and time in British general practice', Social Science and Medicine, 20:7 (1985), 659-66.

24 J. M. Wilks, 'Diabetes - a disease for general practice', JRCGP, 23:126 (1973), 46-54.

25 Ibid., p. 52.

26 On the importance of documentary technologies for maintaining visibility, and thus discipline: Foucault, Discipline and Punish, pp. 189-92.

27 G. Horobin and J. McIntosh, 'Time, risk and routine in general practice', Sociology of Health and Illness, 5:3 (1983), 312-31.

28 M. T. Wojciechowski, 'Systematic care of diabetic patients in a general practice', JRCGP, 32:242 (1982), 532.

29 J. D. Grene and J. M. Henderson, 'Automated recall in general practice', JRCGP, 21:107 (1971), 352-5.

30 R. L. Gibbins and J. Saunders, 'Develop diabetic care in general practice', BMJ, 297:6642 (1988), 187-9.

31 B. Hurwitz, C. Goodman, and J. Yudkin, 'Prompting the clinical care of non-insulin dependent (type-II) diabetic patients in an inner-city area: one model of community care', BMJ, 306:6878 (1993), 624-5.

32 Foucault, Discipline and Punish, p. 154.

33 Wilks, 'Diabetes', p. 46.

34 Interview with B. Potts conducted by the author.

35 E. Martin and S. Goodwin, 'Audit of diabetic care', JRCGP, 38:308 (1988), 124.

36 G. F. Morgan, D. A. Cadman, P. H. Edwards, T. C. O’Dowd, and R. Harvard Davis, 'Diabetes care: whose responsibility?', BMJ, 289:6454 (1984), 1310.

37 Gibbins and Saunders, 'Develop diabetic care in general practice', p. 189. 
38 C. Pope, 'Trouble in store: some thoughts on the management of waiting lists', Sociology of Health and Illness, 13:2 (1991), 205-7.

39 T. Benson, 'Why general practitioners use computers and hospitals do not - part 1: incentives', BMJ, 325:7372 (2002), 1086-9; T. Benson, 'Why general practitioners use computers and hospitals do not - part 2: scalability', $B M J, 325: 7372$ (2002), 1090-3. The divergence in computerisation between general practice and hospitals has perhaps been the most striking inequality.

40 T. Pinch and W. E. Bijker, 'The social construction of facts and artifacts: or how the sociology of science and sociology of technology might benefit each other', in Bijker, Hughes, and Pinch (eds.), The Social Construction of Technological Systems, pp. 11-44.

41 B. Hurwitz and J. Yudkin, 'Diabetes care: whose responsibility?', BMJ, 289:6450 (1984), 1000-1.

42 Martin and Goodwin, 'Audit', p. 124.

43 See Chapters 1 and 2.

44 B. M. Doyle, The Politics of Hospital Provision in Early Twentieth-Century Britain (London: Pickering and Chatto, 2014).

45 R. Wall, 'Using bacteriology in elite hospital practice: London and Cambridge, 1880-1920', Social History of Medicine, 24:3 (2011), 776-95; A. Hull, 'Hector's house: Sir Hector Hetherington and the academization of Glasgow hospital medicine before the NHS', Medical History, 45:2 (2001), 232-6.

46 See Chapters 2 and 1 respectively.

47 B. L. Craig, 'The role of records and of record-keeping in the development of the modern hospital in London, England and Ontario, Canada', Bulletin of the History of Medicine, 65:3 (1991), 376-97; J. D. Howell, Technology in the Hospital: Transforming Patient Care in the Early Twentieth Century (Baltimore: Johns Hopkins University Press, 1995).

48 V. Hess and J. A. Mendelsohn, 'Case and series: medical knowledge and paper technology, 1600-1900', History of Science, 48:3 (2010), 304; B. L. Craig, 'Hospital records and record-keeping c.1850-c.1950, part 1: the development of records in hospitals', Archivaria, 29:1 (1989), 61-3. On casebooks: L. Kassell, 'Casebooks in early modern England: medicine, astrology and written records', Bulletin of the History of Medicine, 88:4 (2014), 595-625.

49 Craig, 'The role of records and of record-keeping', pp. 383-8; Howell, Technology in the Hospital, pp. 42-56.

50 Hess and Mendelsohn, 'Case and series', pp. 296-310; Craig, 'The role of records and of record-keeping', pp. 388-93; D. Cantor, 'Introduction: cancer control and prevention in the twentieth century', Bulletin of the History of Medicine, 81:3 (2007), 12-13. 
51 G. Weisz, A. Cambrosio, P. Keating, L. Knaapen, T. Schlich, and V. J. Tournay, 'The emergence of clinical practice guidelines', Milbank Quarterly, 85:4 (2007), 691-727.

52 M. Edwards, Control and the Therapeutic Trial: Rhetoric and Experimentation in Britain, 1918-48 (Amsterdam: Rodopi, 2007), pp. 93-119; M. Berg, 'Problems and promises of the protocol', Social Science and Medicine, 44:8 (1997), 1081-8.

53 D. Cantor, 'The MRC's support for experimental radiology during the inter-war years', in J. Austoker and L. Bryder (eds.), Historical Perspectives on the Role of the MRC: Essays in the History of the Medical Research Council of the United Kingdom and its Predecessor, the Medical Research Committee, 1913-1953 (Oxford: Oxford University Press, 1989), pp. 199-200.

54 Standing Medical Advisory Committee of the CHSC, The Standardization of Hospital Medical Records: Report of the Sub-Committee (London: HMSO, 1965).

55 G. Rivett, From Cradle to Grave: Fifty Years of the NHS (London: King's Fund, 1998), pp. 96-7, 181-2; T. Osborne, 'Epidemiology as an investigative paradigm: the College of General Practitioners in the 1950s', Social Science and Medicine, 38:2 (1994), 317-26.

56 'Moving towards clinical integration', BMJ, 2:6402 (1976), 964.

57 Edwards, Control and the Therapeutic Trial; S. Sturdy and R. Cooter, 'Science, scientific management and the transformation of medicine in Britain, c.1870-1950', History of Science, 36:4 (1998), 429; C. Lawrence, Rockefeller Money, The Laboratory and Medicine in Edinburgh, 1919-1930: New Science in an Old Country (New York: University of Rochester Press, 2005), p. 269.

58 N. R. Eder, National Health Insurance and the Medical Profession in Britain, 1913-39 (London: Garland, 1982), pp. 155-62.

59 S. Taylor, Good General Practice: A Report on a Survey (London: Oxford University Press, 1954), pp. 147-64, esp. pp. 148-9.

60 Ibid.; Standing Medical Advisory Committee of the CHSC, The Standardization of Hospital Medical Records.

61 Ibid.

62 TNA, MH 160/416, letter by Dr John Bingle to multiple recipients (including Dr Jarrett, Dr Pyke, and Dr Montgomery), untitled, 9 December 1968, p. 1.

63 TNA, MH 160/416, letter by Dr John Bingle to Dr Avery Jones (DHSS), untitled, 5 January 1969 , p. 5.

64 TNA, MH 160/416, H/H.183/29, letter by Dr John Bingle to Mr Alderman (DHSS), 'Advisory committee on hospital medical records', 5 July 1969, p. 1.

65 See Chapter 1. 
66 On the ways 'non-human' objects order action and knowledge: B. Latour, Reassembling the Social: An Introduction to Actor-Network-Theory (Oxford: Oxford University Press, 2005), pp. 70-85.

67 Albuminuria is the passing of protein into the urine, indicating kidney disease.

68 Howell, Technology in the Hospital, pp. 42-56.

69 A. P. Kratky, 'An audit of the care of diabetics in one general practice', JRCGP, 27:182 (1977), 536-43, esp. pp. 539-40.

70 Ibid., p. 540.

71 Ibid.

72 Ibid., p. 541.

73 Ibid., p. 540.

74 Ibid., p. 541.

75 M. Berg, 'Practices of reading and writing: the constitutive role of the patient record in medical work', Sociology of Health and Illness, 18:4 (1996), 499-524.

76 RCGP, Diabetes Clinical Information Folder (London: RCGP, 1988).

77 C. Waine, Why Not Care for your Diabetic Patients?, 2nd edition (London: RCGP, 1988), p. 2.

78 D. Irvine, A Doctor's Tale: Professionalism and Public Trust (Abingdon: Radcliffe Medical Press, 2003), pp. 11-21.

79 N. Bosanquet and C. Salisbury, 'The practice', in I. Loudon, J. Horder, and C. Webster (eds.), General Practice under the National Health Service, 1948-1997 (Oxford: Oxford University Press, 1998), p. 53, appendix D.1.

80 J. T. Corbett, 'Keeping records in general practice', JCGP, 5:2 (1962), 270-1.

81 See Chapter 2.

82 C. E. Upton, 'Diabetic community care', The Practitioner, 215:1284 (1975), 83-6.

83 Ibid., p. 83.

84 R. D. Hill, 'Community care service for diabetics in the Poole area', BMJ, 1:6018 (1976), 1138.

85 P. Home and S. Walford, 'Diabetes care: whose responsibility?', BMJ, 289:6447 (1984), 713-14.

86 Hill, 'Community care service for diabetics', p. 1138.

87 Marking a comparative weakness in disciplinary relationships: Foucault, Discipline and Punish.

88 TNA, MH 150/953, report, 'The Community Care Service (CCS) for diabetics in the Poole area', 1983, pp. 10-12.

89 Hurwitz and Yudkin, 'Diabetes care'.

90 J. Day, H. Humphries, and H. Alban-Davies, 'Problems of comprehensive shared diabetes care', BMJ, 240:6587 (1987), 1591.

91 Waine, Why Not Care for your Diabetic Patients?, p. 28. 
92 S. Ezedum and D. Kerr, 'Collaborative care of hypertensives, using a shared record', BMJ, 2:6099 (1977), 1402.

93 On NHS and research: H. Valier and C. Timmermann, 'Clinical trials and the reorganization of medical research in post-Second World War Britain', Medical History, 52:4 (2008), 493-510; Hull, 'Hector's house', pp. 236-40.

94 'Moving towards clinical integration', p. 964.

95 Hill, 'Community care service for diabetics', p. 1138.

96 Day et al., 'Problems of comprehensive shared diabetes care', pp. 1590-1.

97 Gibbins and Saunders, 'Develop diabetic care in general practice.'

98 'Diabetes: a protocol', in RCGP, Diabetes Clinical Information Folder, appendix 4.

99 J. C. Scott, Seeing Like a State: How Certain Schemes to Improve the Human Condition Have Failed (New Haven: Yale University Press, 1998).

100 R. Pinder, R. Petchey, S. Shaw, and Y. Carter, 'What's in a care pathway? Towards a cultural cartography of the new NHS', Sociology of Health and Illness, 27:6 (2005), 759-79.

101 'Diabetes: a protocol', in RCGP, Diabetes Clinical Information Folder, appendix 4, p. 8.

102 See Chapters 5 and 6 for the quantification and codification of good care after the mid-1980s.

103 See the box in Fig. 3.2 suggesting that patients 'Joi[n] the Diabetic Association'.

104 P. Day, R. Klein, and F. Miller, A Comparative US-UK Study of Guidelines (London: Nuffield Trust, 1998), pp. 13-14, 19.

105 C. O. Hawthorne, 'The freedom of medicine', BMJ, 2:3432 (1926), 705-8, esp. p. 706; C. Lawrence, 'Incommunicable knowledge: science, technology and the clinical art in Britain, 1850-1914', Journal of Contemporary History, 20:4 (1985), 503-20.

106 Foucault, Discipline and Punish. As was the case for patients: Sinding, 'Flexible norms?'

107 These were tools for cultivating the self-managing self: D. Willems, 'Managing one's body using self-management techniques: practicing autonomy', Theoretical Medicine and Bioethics, 21:1 (2000), 23-38.

108 Kratky, 'An audit of the care of diabetics in one general practice', pp. 536-7.

109 B. Essex, 'Records and audit', in RCGP, Clinical Information Folder, appendix 19, p. 1.

110 Foucault, Discipline and Punish.

111 'A medical audit', The Lancet, 280:7251 (1962), 339-40; I. McWhinney, 'Medical audit in North America', BMJ, 2:5808 (1972), 277-9; H. W. K. Acheson, 'Medical audit and general practice', The Lancet, 305:7905 (1975), 511-13. There were differences in aims and contexts, however: W. J. Jackson, A. S. Paterson, C. K. M. Pong, and S. Scarparo, 'Doctors under 
the microscope: the birth of medical audit', Accounting History Review, 23:1 (2013), 23-47.

112 For instance, in the teaching profession: F. S. E. Hatfield, 'Monitoring general practitioners', JRCGP, 26:171 (1976), 764.

113 M. Power, The Audit Society: Rituals of Verification (Oxford: Oxford University Press, 1999); A. Digby, The Evolution of British General Practice, 1850-1948 (Oxford: Oxford University Press, 1999).

114 Armstrong, 'Space and time in British general practice', p. 662; P. Sleight, J. A. Spencer, and E. W. Towler, 'Oxford and McKinsey: Cogwheel and beyond', BMJ, 1:5697 (1970), 682-4; B. L. E. Reedy, 'Morale and management in general practice', JRCGP, 16:1 (1968), 3-11.

115 T. Osborne, 'Power and persons: on ethical stylisation and personcentred medicine', Sociology of Health and Illness, 16:4 (1994), 529; J. N. Morris, Uses of Epidemiology, 2nd edition (London: E. \& S. Livingstone, 1964); A. L. Cochrane, Effectiveness and Efficiency: Random Reflections on the Health Services (London: Nuffield Provincial Hospitals Trust, 1972).

116 B. J. Doney, 'An audit of the care of diabetics in a group practice', JRCGP, 26:171 (1976), 734-2, esp. p. 734.

117 Kratky, 'An audit of the care of diabetics in one general practice', p. 536.

118 Ibid., pp. 536-43.

119 Doney, 'An audit of the care of diabetics'.

120 D. R. R. Williams, C. Munroe, C. J. Hospesdales, and R. H. Greenwood, 'A three-year evaluation of the quality of diabetes care in the Norwich community care scheme', Diabetic Medicine, 7:1 (1990), 74-9.

121 T. M. Hayes and J. Harries, 'Randomised controlled trial of routine hospital clinic care versus routine general practice care for type-II diabetics', BMJ, 289:6447 (1984), 728.

122 J. S. Yudkin, B. J. Boucher, K. E. Schopflin, B. T. Harris, H. R. Claff, N. J. D. Whyte, B. Taylor, D. H. Mellins, A. B. Wootliff, J. G. Safir, and E. J. Jones, 'The quality of diabetic care in a London health district', Journal of Epidemiology and Community Health, 34:4 (1980), 277.

123 Valier and Bivins, 'Organization, ethnicity and the British National Health Service'.

124 P. J. Burrows, P. J. Gray, A.-L. Kinmonth, D. J. Payton, G. A. Walpole, R. J. Walton, D. Wilson, G. Woodbine, 'Who cares for the patient with diabetes? Presentation and follow-up in seven Southampton practices', JRCGP, 37:295 (1987), 65.

125 D. R. R. Williams, P. D. Home, and Members of a Working Group of the Research Unit of the RCP and BDA, 'A proposal for continuing audit of diabetes services', Diabetic Medicine, 9:8 (1992), 759-64, esp. p. 759.

126 Marks, The Progress of the Experiment.

127 P. A. Thorn and R. G. Russell, 'Diabetic clinics today and tomorrow: mini-clinics in general practice', BMJ, 2:5865 (1973), 534-6; B. Singh, 
M. Holland, and P. Thorn, 'Metabolic control of diabetes in general practice clinics: comparison with a hospital clinic', BMJ, 289:6447 (1984), $726-8$.

128 Williams et al., 'A three-year evaluation of the quality of diabetes care in the Norwich community care scheme'.

129 Day et al., 'Problems of comprehensive shared diabetes care'.

130 E. Wilkes and E. E. Lawton, 'The diabetic, the hospital and primary care', JRCGP, 30:213 (1980), 199-206.

131 Keating and Cambrosio, 'Cancer clinical trials'.

132 Gibbins and Saunders, 'Develop diabetic care in general practice', p. 189.

133 For the long history of distinguishing between testing and interpretation: S. J. Reiser, Medicine and the Reign of Technology (Cambridge: Cambridge University Press, 1978).

134 C. Bradshaw and J. Spencer, 'Nurse-run diabetic clinics in general practice', Diabetic Medicine, 7:7 (1990), 572-3.

135 T. J. Kemper and S. R. Hayter, 'Audit of diabetes in general practice', BMJ, 302:6774 (1991), 451-3, esp. p. 452.

136 Ibid., p. 453.

137 N. Black and E. Thompson, 'Obstacles to medical audit: British doctors speak', Social Science and Medicine, 36:7 (1993), 849-56.

138 Interview with M. MacKinnon conducted by the University of Oxford, 23 April 2007, available at: www.diabetes-stories.com/interview.asp? $\mathrm{UID}=62$ (accessed April 2017).

139 M. MacKinnon, R. M. Wilson, C. A. Hardisty, and J. D. Ward, 'Novel role for specialist nurses in managing diabetes in the community', BMJ, 299:6698 (1989), 552-4.

140 On the role of key individuals working within a broader policy structure: M. Gorsky, "To regulate and confirm inequality”? A regional history of geriatric hospitals under the English National Health Service, c.1948-1975', Ageing \& Society, 33:4 (2013), 610-11.

141 K. Piwernetz, P. D. Home, O. Snorgaard, M. Antsiferov, K. StaehrJohansen, and M. Krans for the DiabCare Monitoring Group of the St Vincent's Declaration Steering Committee, 'Monitoring the targets of the St Vincent Declaration and the implementation of the quality management in diabetes care: the DiabCare initiative', Diabetic Medicine, 10:4 (1993), 374.

142 Williams et al., 'A three-year evaluation of the quality of diabetes care in the Norwich community care scheme. For the 'administrative way of knowing': Sturdy and Cooter, 'Science, scientific management and the transformation of medicine in Britain'. 